undiscovered aroma compounds in Kentucky bourbon as well as old favourites such as whiskylactone.

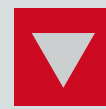

\section{Sippin' nectar}

The fermented nectar of the bertam palm, as drunk by Malaysia's pen-tailed tree shrew, is about as alcoholic as beer. Yet video evidence shows no sign of inebriation. Perhaps they just like the taste...

\section{ON THE RECORD} “Dr Mitchell is a great American, but we do not share his opinions on this issue."

NASA's response to Edgar Mitchell's claim that it is covering up contact with alien civilizations. Mitchell was a member of the Apollo 14 crew.

\section{NUMBER CRUNCH \\ $\mathbf{7 7} \%$ of Americans accepted that there is solid evidence for global warming in January 2007.}

$71 \%$ were of that opinion in April 2008.

$13 \%$ was the drop in the acceptance rate among Republicans.

$2 \%$ was the drop among Democrats.

\section{WORDWATCH}

\section{LOOSE}

The Stanford Linear Accelerator Center may need a new name, because Stanford University will not let the government copyright its current one. An elegantly synonymic suggestion from a SLAC website: the Linearly Organised Optimum Science Establishment.

During August, Sidelines will be off on its summer holiday.

\title{
Thousands of proteins affected by miRNAs
}

MicroRNAs (miRNAs) subtly influence a vast number of proteins involved in most key biological processes, according to the first large-scale analyses of how these small pieces of noncoding RNA affect proteins (M. Selbach et al. Nature doi:10.1038/nature07228; 2008; D. Baek et al. Nature doi:10.1038/nature07242; 2008).

"These papers represent a tour de force in miRNA research, utilizing state-of-the-art technology to tackle a very vexing problem in biology, namely the identification of miRNA target genes," says Frank Slack, a molecular biologist and miRNA researcher at Yale University.

The studies could help answer long-standing questions about how miRNAs work. These tiny snippets of genome, just 21 to 25 nucleotides long, were once thought to be 'junk' material because they are not translated into protein. In fact, in animals they control protein levels through two mechanisms: by breaking down the messenger RNA 'read-out' from a gene; and by stopping the messenger RNA from being translated into protein. But previous studies had taken only a global look at how miRNAs affect messenger RNA, because of the technical difficulties in examining how miRNAs affect thousands of proteins at once.

Two teams of scientists have now tackled this problem using a state-of-the-art version of mass spectrometry. The technique, called SILAC (stable isotope labelling with amino acids in cell culture), relies on the use of heavy isotopes to label proteins as they are being produced by cells. The researchers used the isotopes to label thousands of proteins being made in cells that had been forced to overexpress or downregulate certain miRNAs. They then compared labelled protein levels in these cells with levels of corresponding proteins in control cells. One of the teams, co-led by Matthias Selbach and Nikolaus Rajewsky of the Max Delbrück Center for Molecular Medicine in Berlin, modified the SILAC method to label and compare proteins being made by treated and control cells, so that their results would not be skewed by long-lived proteins.

These analyses showed that a single miRNA can indeed dampen levels of hundreds of proteins by impeding their translation - not just by breaking down their messenger RNAs. The studies also examined whether the proteins' levels changed in ways that had been predicted by computer algorithms and found that the algorithms varied in their predictive abilities.

But the most intriguing finding from the studies is that the effects of miRNAs on proteins are usually quite modest, changing their expression levels by less than twofold. That almost led Steven Gygi, a protein chemist at Harvard Medical School in Boston, Massachusetts, to scotch one of the studies after he used a mass spectrometer to examine the first batch of miRNA-boosted cells. "The experimental data looked just like the controls, so we thought it hadn't worked," says Gygi.

He told his collaborator, David Bartel, a molecular biologist and Howard Hughes investigator at the Whitehead Institute for Biomedical Research in Cambridge, Massachusetts, that he wanted to end the project. But Bartel was undeterred: "After working on miRNAs for seven years, we've learned that the data are very subtle," says Bartel.

A deeper statistical analysis proved Bartel right - the effects were there, but they were often mall. Both teams confirmed their results by using knocked-out miRNAs in animal cells.

But although most miRNA effects may be small, they can still be powerful, according to the Delbrück group. The team found one miRNA, 'let-7b', that can change production of a key protein, called Dicer, by as much as fourfold. Dicer is involved in making all miRNAs and silencing RNAs (siRNAs), which shut down genes through the process of RNA interference. So let-7b directly or indirectly controls the expression of thousands of genes - a finding that wouldn't have been seen if the team had looked only at Dicer's messenger RNA, because most of let-7b's effects on Dicer occur at the translational level, the team found.

The data paint a picture of miRNAs as regulators that fine-tune protein expression in complex and overlapping patterns. That could complicate the picture for drug developers, because most miRNAs have so many different targets. But this is not necessarily a bad thing, as Selbach points out, because some of the most effective drugs hit multiple molecular targets. "I think it's a very beautiful finding, because it shows that the system is very robust," Selbach says.

Erika Check Hayden 